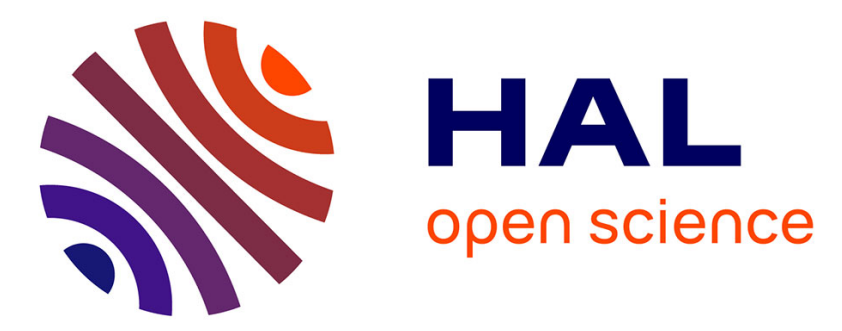

\title{
Actualités en allergologie alimentaire et pédiatrique
}

Amandine Divaret-Chauveau

\section{To cite this version:}

Amandine Divaret-Chauveau. Actualités en allergologie alimentaire et pédiatrique. Revue francaise d'allergologie, 2019, 59 (3), pp.232-234. 10.1016/j.reval.2019.02.208 . hal-03486365

\section{HAL Id: hal-03486365 https://hal.science/hal-03486365}

Submitted on 20 Dec 2021

HAL is a multi-disciplinary open access archive for the deposit and dissemination of scientific research documents, whether they are published or not. The documents may come from teaching and research institutions in France or abroad, or from public or private research centers.
L'archive ouverte pluridisciplinaire HAL, est destinée au dépôt et à la diffusion de documents scientifiques de niveau recherche, publiés ou non, émanant des établissements d'enseignement et de recherche français ou étrangers, des laboratoires publics ou privés.

\section{다)(1) $\$$}

Distributed under a Creative Commons Attribution - NonCommerciall 4.0 International 


\section{Actualités en allergologie alimentaire et pédiatrique}

Current developments in food and paediatric allergy

Amandine Divaret-Chauveau* ${ }^{1,2,3}$

${ }^{1}$ Unité d'allergologie pédiatrique, Hôpital d'enfants, CHRU de Nancy, Vandoeuvre les Nancy

${ }^{2}$ EA 3450 DevAH, Faculté de Médecine, Université de Lorraine, Vandoeuvre les Nancy

${ }^{3}$ UMR 6249 Chrono-Environnement, Université de Bourgogne Franche Comté, Besançon

Auteur correspondant :

Amandine Divaret-Chauveau

Unité d'allergologie pédiatrique,

Hôpital d'enfants, CHRU de Nancy,

Rue du Morvan,

54511 Vandoeuvre les Nancy

a.chauveau@chru-nancy.fr

Tél : 0383155230 / Fax : 0383154686 
Mots clés : aliments ; allergie ; enfant ; prévalence ; traitement

Keywords: food; allergy; children; prevalence; treatment

\section{Prévalence}

L'étude australienne HelathNuts fournit des données robustes sur la prévalence des allergies alimentaires (AA) confirmées par test de provocation orale (TPO) à l'âge de 6 ans sur plus de 5000 enfants en population générale. A l'âge d' 1 an, $3 \%$ avaient une AA à l'arachide, seuls $18,5 \%$ des enfants consommaient des fruits à coques (fac) et la prévalence d'AA aux fac rapportée par les parents était de $0,1 \%$. A l'âge de 6 ans, la prévalence d'AA aux fac était de $3,3 \%$ avec $2,7 \%$ d'AA à la noix de cajou. Plus d'un tiers des enfants sensibilisés aux fac à 1 an avaient une AA aux fac à 6 ans. Parmi les 147 enfants allergiques à l'arachide à 6 ans, 45\% avaient au moins une AA aux fac (36,7\% à la noix de cajou). Dans une enquête similaire chez les enfants de 10 à 14 ans, la prévalence d'AA confirmée par TPO était de 4,5\% avec en tête de liste l'arachide $(2,7 \%)$ et les fac $(2,3 \%)$.

Aux États-Unis, une cohorte rétrospective met en évidence une augmentation des anaphylaxies de l'enfant de 117\% entre 2004 et 2016 (2,3 vs 5 pour 1000 personnes années) et une augmentation des anaphylaxies alimentaires de $177 \%$ (86,3 vs 239,2 pour 1000 personnes années) essentiellement due aux fac et céréales.

Une autre étude américaine, qui a interrogé 40443 adultes, retrouve une prévalence d'AA hautement probable de 10,8\%. Les allergènes les plus fréquents étaient les crustacés, le lait, l'arachide, les fac, le poisson avec $45,3 \%$ de polyallergies. Plus de la moitié des adultes ayant une AA ont eu au moins une réaction allergique sévère, 38,3\% ont eu recours au moins une fois aux services des urgences mais seulement $24,0 \%$ rapportait une prescription d'épinephrine.

\section{Facteurs de risque et prévention}

Une étude récente retrouve une association significative entre la concentration urinaire de phthalates chez la mère durant la grossesse et risque d'asthme chez l'enfant à 7 ans.

L'impact de l'allaitement maternel sur le risque allergique de l'enfant reste controversé. Les travaux de la cohorte EDEN montre que les composants immuns présents dans le lait maternel 
varient selon de nombreux facteurs maternels et environnementaux, rendant l'analyse de l'allaitement maternel comme une seule entité impossible [1].

L'alimentation des premières années de vie a fait l'objet de plusieurs études. Alors que l'introduction précoce de l'arachide et de l'œuf chez les enfants à risque atopique est aujourd'hui recommandée par de nombreuses sociétés savantes, il semble encore difficile d'appliquer ces recommandations à toutes les populations notamment celles avec une faible prévalence d'AA et la question de l'introduction précoce d'autres allergènes fréquents comme les fac reste ouvert. Au sein de la cohorte européenne PASTURE, la consommation d'une grande diversité de fromages à l'âge de 18 mois était associée à une diminution du risque d'asthme et de dermatite atopique à 6 ans [2]. Dans cette même cohorte, les enfants avec des taux élevés de butyrate dans les selles à 1 an (taux liés au régime alimentaire) avaient significativement moins de maladies atopiques à 6 ans [3].

Par ailleurs, la transplantation d'organe semble entraîner une dérégulation immunitaire : une étude canadienne rapporte que $34 \%$ des enfants développent une maladie allergique ou autoimmune après transplantation.

De nombreuses études s'intéressent au lien entre maladies digestives allergiques non IgE médiées (SEIPA, œsophagite à éosinophiles $(\mathrm{OE})$ ) et allergies IgE médiées. Avoir une polyallergie alimentaire semble être le facteur de risque le plus important de développer une OE, maladie qui semble s'inscrire comme la $5^{\text {ème }}$ manifestation de la marche atopique [4].

\section{Allergènes}

L'allergie à l'amande est rare mais la sensibilisation à ce fac est fréquente. L'équipe de l'hôpital du Mont Sinaï a réalisé une étude rétrospective sur 400 enfants ayant bénéficié d'un TPO à l'amande. Parmi ces enfants sensibilisés à l'amande, seuls $4 \%$ avaient une AA à l'amande confirmée et $0,5 \%$ ont présenté une réaction nécessitant une injection d'adrénaline. Bien qu'une sensibilisation importante était corrélée à la positivité du TPO, le pourcentage de TPO négatifs étaient de $81,8 \%$ en cas de prick-test supérieur ou égal à $9 \mathrm{~mm}$ et de $86,4 \%$ en cas d'IgE spécifiques supérieures à 10 kU/L.

Une récente étude européenne a étudié les allergènes de la noix. Parmi 91 personnes allergiques à la noix, 77\% présentaient des réactions sévères (100\% chez les moins de 14 ans). Les réactions sévères étaient associés à un taux d’IgE spécifiques plus élevé pour la noix, rJug r1, nJug r 4 et les 2 fractions vicillines mais il n'y avait pas de différence pour Jug r3 (LTP) et 
Jug r5 (PR-10). Il semble par ailleurs exister une réactivité croisée importante entre l'albumine $2 \mathrm{~S}$ de la noix, rJug r1, et les allergènes de la noisette r Cor a9 et a14.

Une étude sur le processus alimentaire du soja montre une diminution de l'allergénicité du soja après traitement combinant chaleur et pression. Ce traitement entraine une fragmentation des protéines du soja en dehors des globulines $7 \mathrm{~S}$ qui restent intactes.

L'AA aux viandes via l'allergie à l'alpha-gal est une AA de découverte récente avec peu d'études disponibles sur la valeur des tests. La probabilité d'AA à l'alpha-gal est de $95 \%$ avec un taux d'IgE spécifiques alpha-gal supérieur à 5,5kU/L.

\section{$\underline{\text { Traitement }}$}

Immunothérapie orale (ITO)

Une étude finlandaise a suivi sur une période de 11 ans près de 300 enfants ayant bénéficié d'une ITO au lait de vache pour une AA IgE-médiée persistante à l'âge de 5 ans [5]. Un taux d'IgE spécifiques lait de vache élevé et des réactions allergiques en début d'ITO étaient des facteurs prédictifs d'échec et d'anaphylaxie.

L'efficacité de l'ITO au blé a été analysé chez 46 patients ayant une AA au blé persistante (âge médian 8,7 ans). Une ITO à faible dose de blé permet d'obtenir 30\% de désensibilisation à 2 ans et $13 \%$ de tolérance à long terme. Environ $15 \%$ ont présenté une réaction adverse mais seuls 0,08\% des participants ont eu recours à l'adrénaline.

Concernant l'arachide, l'essai Take-Away a inclut 77 enfants âgés de 5 à 15 ans ayant une AA sévère à l'arachide, 57 d'entre eux bénéficie d'une ITO à forte dose de maintenance pour une durée de 4 ans et les 20 autres enfants restent en éviction. Les premiers résultats de cette étude montrent que seuls $21,1 \%$ des enfants atteignent la dose de maintenance de $5 \mathrm{~g}$ de protéines d'arachide, 54,4\% poursuivent le traitement à une dose de maintenance individualisée (médiane de 2,7g) et 24,5\% arrêtent l'ITO principalement du fait d'un dégoût (67\%) et de réactions adverses (27\%). Près de $20 \%$ des enfants du groupe ITO ont présenté une anaphylaxie vs 0 dans le groupe éviction. Il semble que l'importance de la dose de maintenance soit corrélée au nombre, à la sévérité des réactions adverses et aux taux d'arrêt. Les résultats à long terme de cette étude nous permettront d'en savoir plus sur l'importance de la durée de l'ITO. 
La prise de décision partagée entre le médecin et le patient se développe en allergologie [6]. Avec l'émergence de l'ITO pour le traitement des AA, il serait aussi utile de développer des outils de prise de décision partagée adaptés à cette thérapeutique.

Omalizumab (OMZ)

L'impact de l'OMZ sur les comorbidités atopiques à 1 an de l'initiation du traitement a été étudié chez plus de 200 patients présentant un asthme sévère. Le traitement avait un effet positif sur la rhinite allergique dans $82,2 \%$ des cas, sur la dermatite atopique dans $82,1 \%$ des cas et sur les AA dans 67,3\% des cas. Même si l'OMZ n'est pas recommandé dans le traitement des AA sévères, cette étude ainsi que des descriptions de cas montrent une efficacité notable l'OMZ sur les AA sévères.

\section{Références}

[1] Berdi M, de Lauzon-Guillain B, Forhan A, Castelli FA, Fenaille F, Charles M-A, et al. Immune components of early breastmilk: association with maternal factors and with reported food allergy in childhood. Pediatr Allergy Immunol 2018. doi:10.1111/pai.12998.

[2] Nicklaus S, Divaret-Chauveau A, Chardon M-L, Roduit C, Kaulek V, Ksiazek E, et al. The protective effect of cheese consumption at 18 months on allergic diseases in the first 6 years. Allergy 2018. doi:10.1111/all.13650.

[3] Roduit C, Frei R, Ferstl R, Loeliger S, Westermann P, Rhyner C, et al. High levels of Butyrate and Propionate in early life are associated with protection against atopy. Allergy 2018. doi:10.1111/all.13660.

[4] Hill DA, Grundmeier RW, Ramos M, Spergel JM. Eosinophilic Esophagitis Is a Late Manifestation of the Allergic March. J Allergy Clin Immunol Pract 2018;6:1528-33. doi:10.1016/j.jaip.2018.05.010.

[5] Kauppila TK, Paassilta M, Kukkonen AK, Kuitunen M, Pelkonen AS, Makela MJ. Outcome of oral immunotherapy for persistent cow's milk allergy from 11 years of experience in Finland. Pediatr Allergy Immunol 2019. doi:10.1111/pai.13025. [6] Blaiss MS, Steven GC, Bender B, Bukstein DA, Meltzer EO, Winders T. Shared decision making for the allergist. Ann Allergy Asthma Immunol 2018. doi:10.1016/j.anai.2018.08.019. 
